\title{
Complementary study of the photoluminescence and electrical properties of ZnO films grown on $4 \mathrm{H}-\mathrm{SiC}$ substrates
}

\author{
V. Khranovskyy ${ }^{1}$, I. Shtepliuk ${ }^{1,2}$, L. Vines ${ }^{3}$ and R. Yakimova ${ }^{1}$ \\ ${ }^{1}$ Department of Physics, Chemistry and Biology (IFM), Linkoping University, Sweden \\ ${ }^{2}$ Frantsevich Institute for Problems of Materials Science NAS of Ukraine, 3 Krzhizhanivsky str., \\ 03680 Kyiv, Ukraine \\ ${ }^{3}$ UIO, Department of Physics, Oslo, Norway
}

\begin{abstract}
We have studied the photoluminescence and electrical properties of $\mathrm{ZnO}$ films grown epitaxially by atmospheric pressure MOCVD on $4 \mathrm{H}-\mathrm{SiC}$ substrates. The dominating $\mathrm{D}^{0} \mathrm{X}$ line on the low temperature PL spectrum is attributed to the emission of an exciton bound to the neutral donor. The intensity of this line correlates with the electrical properties of the films: the decrease of $\mathrm{D}^{0} \mathrm{X}$ intensity occurs simultaneously with the increase of the carrier's mobility. This we explain as donor activation providing free electrons to the conduction band. Based on the comparison of the calculated value of donor binding energy, the literature data and complementary SIMS analysis a suggested donor impurity is aluminum (Al). The exciton localization energy is 16.3 $\mathrm{meV}$, and agrees well with localization energy of $15.3 \mathrm{meV}$ for Al impurity reported by other authors (e.g. Ref. [32]). The thermal activation energy $E_{\mathrm{D}}=20 \mathrm{meV}$, determined from the Hall data and is in agreement with the optical activation energy $\sim 20 \mathrm{meV}$, which is derived from the temperature-dependent PL study. The calculated value of the donor binding energy of $54.3 \mathrm{eV}$ is in agreement with the ionization energy of $53 \mathrm{meV}$ mentioned in earlier reports for $\mathrm{Al}$ in $\mathrm{ZnO}$ films. Our results prove that the commonly observed line at $\sim 3.3599 \mathrm{eV}$ on low temperature PL spectra of $\mathrm{ZnO}$ is a neutral donor bound exciton emission due to the $\mathrm{Al}$ impurity.
\end{abstract}

Keywords: photoluminescence, zinc oxide, mobility, epitaxy, silicon carbide

Corresponding author: Ivan Shtepliuk, Linköping University, Department of Physics, Chemistry, and Biology (IFM), 58381 Linköping, Sweden, phone: 0762644554, e-mail: ivan.shtepliuk@liu.se

\section{Introduction}

The interest to $\mathrm{ZnO}$ as semiconductor material for optoelectronics is mainly driven due to its wide direct band gap $(\sim 3.3 \mathrm{eV})$, large exciton binding energy $(\sim 60 \mathrm{meV})$ and possibility of the band-gap engineering via alloying with $\mathrm{CdO}, \mathrm{MgO}$ and $\mathrm{BeO}$ [1-5]. The latter has attracted special attention for elaboration of bright light emitters in the blue/ultraviolet (UV) spectral region due to strong quantum confinement predicted for $\mathrm{ZnO}$-based quantum wells (QWs) [6-8]. 
The light emission processes are until large extent governed by the structural quality of the $\mathrm{ZnO}$ layers, directly determining the efficiency of the radiative recombination channels (RRC). It is a common knowledge that the realization of the optoelectronic devices demand (i) using the highquality strain-free barrier and well layers, (ii) availability of the reliable technology of the both types of conductivity and, the last but not least, (iii) clear understanding the role of unintentional dopants in forming the RRC and carrier transport. Because of well-known problem with obtaining the $p$-type $\mathrm{ZnO}$ it is very important to develop the alternative technology through the utilizing foreign substrates of intrinsic $p$-type conductivity, promoting the growth strain-free $n$ type $\mathrm{ZnO}$ films with understandable characteristics. Nevertheless, in most cases the lattice mismatch between the foreign substrates and $\mathrm{ZnO}$ films can give rise to a large interfacial strain between epilayer and substrate and can be a reason for a high defect density in the $\mathrm{ZnO}$ epitaxial layers [9]. In the light of this obstacle the most appropriate interface for $\mathrm{ZnO}$ growth can become silicon carbide ( $\mathrm{SiC}$, e.g. $4 \mathrm{H}-\mathrm{SiC}, 6 \mathrm{H}-\mathrm{SiC}$, etc.), which is a mature semiconductor material, available with both $n$ - and $p$-type conductivity. However, the in-plane lattice parameters mismatch of $\mathrm{SiC}$ and $\mathrm{ZnO}$ is approximately 5\%, resulting in the compressive strain in $\mathrm{ZnO}$ initial layers. The strain can, however, be mitigated and material can release the stress via creating mismatch dislocations and grain boundaries. Therefore, growth of defect-free high quality $\mathrm{ZnO}$ on $\mathrm{SiC}$ is a motivating scientific task, which requires applying different growth processes and substrate related tricks. One of the possible solutions can be using vicinal SiC substrates -off-cut a several degrees $(0.5,2,4.5,8$, etc. $)$ from their vertical $c$-axis [10]. To our best knowledge, the vicinal surface effect can be responsible for the reduction of the threading defects as reported by Xie et al. [11]. Such substrate usually possesses the steps and terraces on the surface, which can efficiently serve as the nucleation centers for $\mathrm{ZnO}$ atoms. Furthermore, the steps on $\mathrm{SiC}$ surface can serve as the defects introducing points in the controlled and regular way. Earlier, Khranovskyy et al. have demonstrated the growth of $\mathrm{ZnO}$ nanostructures on $\mathrm{p}$-type $\mathrm{SiC}$ substrate [12]. So-called $\mathrm{ZnO}$ nanohexagons were grown in perfect in-plane and out-of-plane epitaxial relationship to the substrate with high quality interface. This, however, is not that straightforward in the case of films or layers growth on such vicinal substrates, where the material relaxation can occur via creating of mismatch dislocation, grain boundaries and other defects. This affects detrimentally structural, optical and electrical properties of $\mathrm{ZnO}$.

Further improvement of the quality of $\mathrm{ZnO}$ films grown on $\mathrm{SiC}$ substrates requires deep understanding a nature of the defects, since they are not only involved into the forming the radiative recombination channels (via creating the electronic states in forbidden band), but also play an important role in electron transport (carrier scattering, donor/acceptor donation). The 
simple way in which one can identify the role of defects is low temperature PL measurement. Nevertheless, the PL spectroscopic fingerprint of $\mathrm{ZnO}$ is very complicated due consisting of multiply overlapping lines. Usually, there are a lot of lines entitled $\mathrm{I}_{1}-\mathrm{I}_{14}$, which are attributed to the certain type of transition [13]. Some lines are associated with the impurities (for example $\mathrm{I}_{4}$ is related to $\mathrm{H}$, whereas $\mathrm{I}_{2}$ is ascribed to $\mathrm{Al}$ etc.). The most common impurities are $\mathrm{H}, \mathrm{C}, \mathrm{Al}, \mathrm{Na}, \mathrm{K}$ etc $[1,2]$ although they differ depending on the material fabrication method. $\mathrm{H}$ and $\mathrm{Al}$ are persistent background donors in $\mathrm{ZnO}$ films, grown by CVD-based techniques. However, it is rather challenging to assign the observed dominating excitonic line to the certain type of impurity, since the energy position of the lines can be affected by the strain/stress in the films, available multiple impurities and interfering intrinsic-defects related phenomena.

In this regard, it is especially interesting to study the thermal-activated processes, which can give direct information about activation energy proper to certain type of the defect and/or process. Furthermore, a symbiotic combination of the complementary electrical and optical studies may provide a better means of assessing the quality of films. From the solid state physics' point of view the coupling of the electrical properties with the PL characteristics can give a clear insight into a problem of optimization of the film's characteristics via fine tuning the exciton population, carrier dynamics and impurity behavior. In this context, our work reinforces the hope of obtaining high-quality zinc oxide films (with controllable and understandable defect subsystem) onto foreign wafers. To our best knowledge, the correlation between temperaturedependent PL characteristics and Hall parameters properties of unintentionally doped $\mathrm{ZnO}$ films onto $\mathrm{SiC}$ substrates with off-cut angle has not been reported so far.

In this work we have deposited the epitaxial films of $\mathrm{ZnO}$ on $4 \mathrm{H}-\mathrm{SiC}$ substrates and characterized them in terms of optical (PL spectroscopy) and electrical properties (Hall and SSRM analysis) at wide temperature range $(10-300 \mathrm{~K})$, complemented additionally by SIMS elemental analysis.

\section{Experimental details}

The $\mathrm{ZnO}$ films were deposited by atmospheric pressure metalorganic chemical vapor deposition (APMOCVD) using the zinc acetylacetonate and oxygen as precursors [14]. The substrate temperature was $550{ }^{\circ} \mathrm{C}$ and the flow rates of Ar, as a buffer gas, and oxygen were 50 and $25 \mathrm{sccm}$, respectively. The commercially available $4 \mathrm{H}-\mathrm{SiC}$ substrates with off-cut angle of $2^{\circ}$ towards the [0001] direction were used as a substrate. The $\mathrm{ZnO}$ growth conditions were optimized for the epitaxial growth of $\mathrm{ZnO}$ films of high crystalline quality, and the structural properties of the identical samples can be found elsewhere [15]. 
The light emission from the $\mathrm{ZnO}$ films was studied by the micro-photoluminescence, carried out in the temperature range $4-290 \mathrm{~K}$ with a frequency doubled Nd:YVO laser as continuous wave excitation source $(\lambda=266 \mathrm{~nm})$. The emitted luminescence was collected and mirrored into a single grating $0.45 \mathrm{~m}$ monochromator equipped with a liquid nitrogen cooled $\mathrm{Si}$ CCD camera with a spectral resolution of about $0.1 \mathrm{meV}$. The excited area was around $10 \mu \mathrm{m}$ in diameter providing an excitation density of $\sim 25 \mathrm{~W} / \mathrm{cm}^{2}$.

TDH Hall measurements were performed in the Van Der Pauw configuration with a probing magnetic field strenght of $1 \mathrm{~T}$ by using a LakeShore 7604. All the measurements were undertaken with the samples in a He bath and dark from $330 \mathrm{~K}$ to the lowest temperature possible where, by ramping the magnetic field at both negative and positive direction, no asymmetries in the measured Hall coefficient were present.

The chemical characterization was carried out by SIMS using a Cameca IMS $7 \mathrm{f}$ instrument with a primary beam of $10 \mathrm{keV} \mathrm{O}_{2}{ }^{+}$or $15 \mathrm{keV} \mathrm{Cs}^{+}$ions. The impurity concentrations were calibrated using implanted reference samples. The crater depths were measured with a Dektak 8 stylus profilometer and a constant erosion rate as a function of sputtering time was assumed.

For Scanning Spreading Resistance Microscopy (SSRM) measurements the samples were cleaved with cross sections (edges) such that electrical resistance profiles could be determined employing a Veeco D3100 atomic force microscope operated in scanning spreading resistance mode (SSRM). A conductive Ti-Pt covered silicon tip was used in contact mode and DC bias voltage was applied between the sample and the tip.

\section{Results and Discussion}

\subsection{Photoluminescence of $\mathrm{ZnO}$ films}

\subsubsection{Donor bound to free exciton transitions analysis of temperature dependent PL spectra}

The temperature dependent PL spectra ranged at 3.6 - 2.6 eV demonstrated intense near band edge (NBE) emission and absence of any emission in the visible range (Fig. 1a). Detailed analysis of the NBE emission revealed the presence of free exciton (FX) and neutral donor bond exciton $\left(\mathrm{D}^{0} \mathrm{X}\right)$ emission bands from the high energy side, followed by two-electron satellite (TES) line and donor-acceptor pair (DAP) emission (Fig. 1b).

In the low-temperature regime, the PL spectrum is dominated by only the lowest energy levels (excitons bound at the shallow neutral donor-type impurity traps), because the thermal population of high-density band levels is very small. These states are characterized by binding energies of the order of a few $\mathrm{meV}$, which is small relative to $k T$ at ordinary temperatures. As can be seen from Figure 1 , the $\mathrm{D}^{0} \mathrm{X}$ emission band (due to the abundance of $\mathrm{D}^{0} \mathrm{X}$ in $\mathrm{ZnO}$ ) is 
dominant in the range of $4 \leq \mathrm{T} \leq 80 \mathrm{~K}$. Furthermore, the weak shoulder in the high-energy side of $\mathrm{D}^{0} \mathrm{X}$ emission band, which is ascribed to the free exciton (FX), is also observed. It is not surprising, since $\mathrm{FX}$ and $\mathrm{D}^{0} \mathrm{X}$ emission bands in $\mathrm{ZnO}$ are commonly overlapped. As the temperature increases above $80 \mathrm{~K}$, the emission of FX becomes prevailing. This is due to the fact that the localized carriers are thermalized to occupy higher energy states.

In other words, as the sample temperature and corresponding thermal energy are increased, excitons dissociate and carriers vacate shallow traps, reducing the intensity of these features in the PL spectrum. At the same time, the band transitions dominate the PL because of the abundance of states taking part (due to the increase in the thermal population of high-energy band levels). Therefore, the temperature-dependent PL spectra in Figure 1 clearly show thermal redistribution among states. The peak labeled FX gains strength with increasing temperature, as expected for thermal population of a higher energy state. In contrast, the peak labeled $\mathrm{D}^{0} \mathrm{X}$ vanishes at temperatures higher than $80 \mathrm{~K}$. Peak $\mathrm{D}^{0} \mathrm{X}$ is strong at low temperature because carriers are trapped at these sites and do not have enough thermal energy to escape, but it disappears at high temperature since the states are sparse relative to the intrinsic bands.

\subsubsection{Features of TES line of temperature dependent PL spectra of $\mathrm{ZnO}$ films}

TES transition occurs when an exciton bound to a neutral donor $\mathrm{D}^{0} \mathrm{X}$ recombines and leaves the donor in an excited state [16]. Therefore, the TES energy is lower than that of the $\mathrm{D}^{0} \mathrm{X}$ peak by the difference in the ground and excited state energies of the neutral donor (as one can see in Fig. 1) [16]. Since the TES radiative transition is a many body quantum process involving impurity, exciton, exciton-impurity electron coupling, and even exciton-phonon coupling [16], the presence/or absence of this line should be correlated with (i) donor-type impurity density influencing on the redistribution of the recombination channels, (ii) crystal quality of the films effecting the density of non-radiative recombination centers and (iii) average rate of excitons involved in radiative processes. This assumption, nevertheless, requires strong experimental verification. In order to understand further the nature of the TES lines for the vicinal films the time-resolved and magneto-optic spectroscopy studies must be carried out [16]. It should be mentioned that the intensity of TES emission decreases with increasing temperature. This trend follows the monotonic change of the intensity of $\mathrm{D}^{0} \mathrm{X}$ emission band with increasing temperature (Fig. 1b). However, this observation is in contrast to the result reported in Ref. [16], where TES emission increases with increasing temperature. These authors revealed that that the TES transition can be associated with a radiative Auger transition rather than a common radiative recombination like the direct radiative recombination of bound excitons [16].

\subsubsection{Donor-acceptor pairs emission in the $\mathrm{ZnO}$ films on 4H-SiC substrate}


The low energy peak ranged at $3.22-3.24 \mathrm{eV}$ is attributed to the donor-acceptor pair (DAP) emission. This emission is ascribed to the optical transition of electron from donor level to acceptor level in the presence of Coulomb and Van der Waal interaction between electrons localized by donor impurities and holes localized by acceptor impurities. In the low temperature regime, a lot of localized carriers are available for forming DAP radiative recombination channels. Therefore, we can see the prominent DAP-related peak on the PL spectra. As temperature increases, the DAP emission quenches due to thermal ionization of the shallow donors and, immediately after, transforms to free electron to acceptor $\left(\mathrm{eA}^{0}\right)$ emission. On one hand, in the high-temperature mode the DAP emission should be blue-shifted by the magnitude of the donor ionization energy. On the other hand, an increase in temperature gives rise to band gap energy shrinkage, leading to red-shift of the DAP/eA ${ }^{0}$ emission peak. In most cases, abovementioned effects compensate each other and, therefore, the energetic position of DAP emission peak is marginally sensitive to the temperature. Naturally, the intensity of this peak sufficiently drops with temperature increasing due to strong thermally-activated carrier delocalization.

\subsubsection{Fitting of the temperature dependent PL spectra}

In the characterization of discrete low-energy states, quantitative analysis of the decrease in PL intensity with temperature can be used to measure the depth of the trap. The integrated PL intensity variation as a function of temperature for the $\mathrm{ZnO}$ films grown on $4 \mathrm{H}-\mathrm{SiC}$ substrate is shown by the Figure 2. Plotting the log of the PL intensity vs. the reciprocal temperature, the slope yields the activation energy for exciting carriers out of the traps. A fit of the experimental data can be performed using the following Arrhenius equation [17]:

$$
I=\frac{I_{0}}{1+C_{1} \exp \left(-E_{a 1} / k_{b} T\right)+C_{2} \exp \left(-E_{a 2} / k_{b} T\right)}
$$

where $k_{b}$ is the Boltzmann constant, $E_{a}$ is the thermal activation energy for thermal quenching, $I_{0}$ is the PL intensity at $0 \mathrm{~K}$ and $C$ is a constant. In this expression, the presence of two states, with activation energies $E_{a 1}$ and $E_{a 2}$, is indicative of two competitive nonradiative recombination channels [18]. The linear fit gives activation energies of about 7.5 and $20 \mathrm{meV}$ for $\mathrm{E}_{\mathrm{a} 1}$ and $\mathrm{E}_{\mathrm{a} 2}$, respectively. The activation energy in low temperature regime indicates that at this range, the PL emission is due to the recombination of $\mathrm{D}^{0} \mathrm{X}$. And at higher temperature, the PL emission is due to the recombination of FX. As for the activation energy of $7 \mathrm{meV}$, we note that it is much smaller than the $13 \mathrm{meV}$ localization energy calculated by Krustok et al.[19], which have pointed out that a low $E_{a}$ value of a few meV may just result from the temperature-dependent capture cross section of the carriers at the recombination centers, and not from a genuine thermal 
activation energy. The estimated activation energy in the high temperature regime is similar to the reported value of $25 \mathrm{meV}$ for FX [20]. The thermal quenching energy of $7 \mathrm{meV}$ is similar to the earlier reported value of $6.8 \mathrm{meV}$ for the neutral donor bound to exciton [21].

Fig. 3 shows the PL peak energies $(\mathrm{eV})$ of $\mathrm{FX}$ and $\mathrm{D}^{0} \mathrm{X}$ versus temperature. The $\mathrm{D}^{0} \mathrm{X}$ and $\mathrm{FX}$ peaks red shift monotonically as temperature increases with different parameters. Varshni's empirical equation [22] can be fitted with the data to find the temperature dependence of the $\mathrm{D}^{0} \mathrm{X}$ and FX peak energies [23], shown as solid lines in Fig. 6. Varshni's empirical equation is as follows:

$$
E(T)=E(0)-\frac{\alpha T^{2}}{\beta+T}
$$

where $\mathrm{E}(0)$ is the band gap at the absolute temperature $\mathrm{T}=0 \mathrm{~K} ; \alpha$ and $\beta$ are the Varshni thermal coefficients. The best fit thermal coefficients $\alpha$ and $\beta$ of energy peak versus temperature are 0.71 $\mathrm{meV} / \mathrm{K}$ and $1000 \mathrm{~K}$ for $\mathrm{D}_{0} \mathrm{X}$ and $0.91 \mathrm{meV} / \mathrm{K}$ and $600 \mathrm{~K}$ for $\mathrm{FX}$, which is in agreement with earlier reported data [21].

An analysis of the results in Fig. 3 also demonstrates that thermal distributions occur in the PL line width of the FX transitions. Figure 4 shows the full width at half maximum (FWHM) of the FX emission line as a function of temperature for $\mathrm{ZnO}$ films grown on the vicinal surface. The FWHM can be described by [24-26]

$$
F W H M(T)=\Gamma_{0}+\gamma_{p h} T+\Gamma_{L O} /\left[\exp \left(\hbar \omega_{L O} / k T\right)-1\right]+\Gamma_{e x} \exp \left(-E_{a} / k T\right)
$$

where $\Gamma_{0}$ is the intrinsic linewidth at $0 \mathrm{~K}$ arising due to electron-electron interaction, impurity, and dislocation, $\gamma_{\mathrm{ph}}$ is electron-acoustic phonon coupling constant, $\Gamma_{\mathrm{ex}}$ is an impurity related factor, and $E_{a}$ is the activation energy averaged over all impurities and $\Gamma_{\mathrm{LO}}$ is the strength of electron-LO phonon interaction [27]. The LO phonon energies $\hbar \omega_{\mathrm{LO}}$ for $\mathrm{ZnO}$ film was taken as $70 \mathrm{meV}[28,29]$. The fitted values for $\mathrm{ZnO}$ are $\Gamma_{0}=19 \mathrm{meV}, \gamma_{\mathrm{ph}}=0.095 \mathrm{meV} / \mathrm{K}, \Gamma_{\mathrm{LO}}=308 \mathrm{meV}$, $\Gamma_{\mathrm{ex}}=250$ and $\mathrm{E}_{\mathrm{a}}=2307 \mathrm{meV}$. The FWHM increases from $28 \mathrm{meV}$ at $80 \mathrm{~K}$ to $66 \mathrm{meV}$ at $290 \mathrm{~K}$. At low temperatures, the linewidth increases with temperature due to the electron-acoustic phonon interaction. The electron-LO phonon interaction and broadening due to impurities and defects scattering become important above $70 \mathrm{~K}$ and eventually dominant at high temperatures for $\mathrm{ZnO}$ films. The obtained results along with reference data are summarized in Table 1.

\begin{tabular}{|c|c|c|c|c|c|c|c|c|c|c|c|c|}
\hline \multirow[t]{2}{*}{ Sample } & \multicolumn{2}{|c|}{$\begin{array}{l}\text { Activation } \\
\text { energy, meV }\end{array}$} & \multicolumn{2}{|c|}{$\begin{array}{l}\text { Thermal } \\
\text { coefficients for } \\
\mathrm{D}_{0} \mathrm{X} \text { line }\end{array}$} & \multicolumn{2}{|c|}{$\begin{array}{c}\text { Thermal } \\
\text { coefficients for } \\
\text { FX line }\end{array}$} & \multirow{2}{*}{$\begin{array}{c}\text { Intrinsic } \\
\text { linewidth } \\
\Gamma_{0} \text { at } 0 \\
\mathrm{~K}, \mathrm{meV}\end{array}$} & \multirow{2}{*}{$\begin{array}{c}\text { Electron- } \\
\text { acoustic } \\
\text { phonon } \\
\text { coupling } \\
\text { constant } \\
\gamma_{\mathrm{ph}}, \\
\mathrm{meV} / \mathrm{K}\end{array}$} & \multirow{2}{*}{$\begin{array}{l}\text { Activation } \\
\text { energy } \\
\text { averaged } \\
\text { over all } \\
\text { impurities } \\
\mathrm{E}_{\mathrm{a}}, \mathrm{meV}\end{array}$} & \multirow{2}{*}{$\begin{array}{c}\text { Impurity } \\
\text { related } \\
\text { factor } \\
\Gamma_{\mathrm{ex}}\end{array}$} & \multirow{2}{*}{$\begin{array}{c}\text { Strength } \\
\text { of } \\
\text { electron- } \\
\text { LO } \\
\text { phonon } \\
\text { interaction } \\
\Gamma_{\mathrm{LO}}, \mathrm{meV}\end{array}$} & \multirow[t]{2}{*}{ Ref } \\
\hline & $\begin{array}{l}\mathrm{E}_{\mathrm{al}}, \\
\mathrm{meV}\end{array}$ & $\begin{array}{l}\mathrm{E}_{\mathrm{a} 2}, \\
\mathrm{meV}\end{array}$ & $\begin{array}{c}\alpha, \\
\mathrm{meV} / \mathrm{K}\end{array}$ & $\beta, K$ & $\begin{array}{c}\alpha, \\
\mathrm{meV} / \mathrm{K}\end{array}$ & $\beta, \mathrm{K}$ & & & & & & \\
\hline
\end{tabular}

Table 1. Best-fit parameters obtained from analysis of the temperature-dependent PL spectra 


\begin{tabular}{|c|c|c|c|c|c|c|c|c|c|c|c|c|}
\hline $\begin{array}{c}\text { ZnO film } \\
\text { on } 4 \mathrm{H}- \\
\mathrm{SiC}\end{array}$ & 7.5 & 20 & 0.71 & 1000 & 0.91 & 600 & 19 & 0.095 & 2307 & 250 & 308 & $\begin{array}{c}\text { This } \\
\text { work }\end{array}$ \\
\hline $\begin{array}{c}\mathrm{ZnO} \\
\text { nanowires } \\
\text { on Si }\end{array}$ & 5.98 & 30.63 & - & - & - & - & 5 & 0.074 & 31.4 & 256.4 & 0 & {$[26]$} \\
\hline $\mathrm{ZnO}$ film & - & 47 & 0.896 & 920 & 0.92 & 920 & 16 & 0.017 & - & - & 594 & {$[30]$} \\
\hline
\end{tabular}

\subsection{Electrical characterization of $\mathrm{ZnO}$ films}

The temperature dependent Hall Effect measurements indicate that the grown $\mathrm{ZnO}$ films are, in principle, non-degenerate semiconductors, but the weak temperature dependence of the carrier concentration suggests that their energetic state is closed to the limit of the degeneracy. The depth dependent resistance - scanned over the cross-section of ZnO film by SSRM is shown on Fig. 5. The low resistivity region within the first $1 \mu \mathrm{m}$ is associated with the $\mathrm{ZnO}$ layer. After this region, the resistance increases very fast and saturates down to the levels related to lowdoped SiC substrate. It is clearly seen that the resistance of the $\mathrm{ZnO}$ layer is relatively uniform, but with some variations. This is due to the (i) inhomogeneous distribution of impurities, defects and other centers of scattering that limit electric transport as well as (ii) fluctuations in free carrier concentration.

Revealing the correlation between the temperature dependent PL spectra and temperature-dependent Hall data gives a deep insight into the electron transport in $\mathrm{ZnO}$ films. As mentioned in Section 3.1., the increase in temperature leads to thermalization of excitons bound to neutral donor impurities. This process occurs when the thermal energy is close to the localization energy of a bound exciton. As a result of this thermal dissociation the free exciton and donor impurity are generated. The latter can donate an electron in the conduction band, thereby increasing the free carrier concentration. Figure 6 shows the temperature dependence of the carrier density. One can see the slight changes in the free carrier concentration (within the entire temperature range), which increases monotonically settling to the density value of about $1 \cdot 10^{19} \mathrm{~cm}^{-3}$.

The behavior of the free carrier concentration with temperature is evidence of the thermal activation of the donor impurities in the film. Assuming that there are three, at least, shallow donor energy levels within the bandgap, the donor activation energies $\Delta E_{D}$ can be derived by the following charge -balance relation, which is valid for non-degenerate $n$-type semiconductors [31]: 


$$
n+N_{A}=\sum_{i=1}^{k}\left[\frac{N_{d i}}{1+\frac{g_{D i} n T^{-3 / 2}}{N_{C}(1)} \exp \left(\frac{\Delta E_{D i}}{k_{B} T}\right)}\right]
$$

We calculate the temperature dependence of the carrier concentration (with donor densities $N_{\mathrm{D} 1}=3 \cdot 10^{18} \mathrm{~cm}^{-3}, \quad N_{\mathrm{D} 2}=2 \cdot 10^{18} \mathrm{~cm}^{-3}, N_{\mathrm{D} 3}=1 \cdot 10^{18} \mathrm{~cm}^{-3}$, and activation energies $\Delta E_{\mathrm{D} 1}=20 \mathrm{meV}$, $\left.\Delta E_{\mathrm{D} 2}=5 \mathrm{meV}, \Delta E_{\mathrm{D} 3}=1 \mathrm{meV}\right)$. As can be seen from the Figure 6 , the fits are best in the high temperature range, suggesting that the corresponding carriers are in the conduction band. At the same time, one can see that the low temperature fits are unacceptable. Probably the single band conduction approach that we used for calculation is not appropriate to explain the low temperature behavior of the carrier concentration in our $\mathrm{ZnO}$ films and is valid only in high temperature regime. The activation energy $\Delta E_{\mathrm{D} 1}$ might be ascribed to transitions from donors to conduction bands, whereas the activation energies $\Delta E_{\mathrm{D} 2}$ and $\Delta E_{\mathrm{D} 3}$ may not be associated with excitation of electrons into the conduction band but it can be referred to the impurity/defect band. It is reasonable to assume that in the low temperature regime only small part of the shallow donors with low activation energies $\Delta E_{\mathrm{D} 2}$ and $\Delta E_{\mathrm{D} 3}$ can donate electrons. An increase in temperature leads to thermal activation of the main part of $\mathrm{Al}$ donors, therefore one can observe more pronounced carrier concentration enhancement. Our results suggest that Al-related donor centers with activation energy $\sim 20 \mathrm{meV}$ mainly contribute to the carrier concentration enhancement with increasing temperature. At the same time, the weak temperature dependence of the carrier concertation in the low temperature mode can be related to the thermal activation of the shallow donors within the impurity/defect band. We believe that only small part of these shallow donors can donate electrons to the conduction band, while the larger part of the excited carrier are probably trapped and do not contribute to the conductivity. According to our estimations, the grown films were characterized by quite low values of the acceptor density $\sim 10^{17} \mathrm{~cm}^{-3}$ and compensation ratio $N_{\mathrm{a}} / N_{\mathrm{d}}$. The temperature dependence of the compensation ratio is depicted on the figure $6 \mathrm{~b}$. As we can see the increase in temperature give rise to decrease in the compensation ratio, suggesting that the thermal activation of aluminum donors in the high temperature regime is responsible for the increase in carrier concentration. The value of the activation energy of $20 \mathrm{meV}$ correlates well with the optical activation energy (which was determined from the temperature dependence of photoluminescence). It should also be noted that the sharp increase in concentration may be due to the temperature dependence of the compensation ratio between the acceptor and donor impurities. In this regard, the results of Hall measurements correlate with results of the temperature dependence of photoluminescence. In 
particular, a significant decrease in the ratio between the integrated intensity of $\mathrm{D}^{0} \mathrm{X}$ excitonic line (related to recombination processes involving neutral donor impurities) to the integrated intensity of the band edge emission was found. Temperature increasing causes thermal dissociation of $\mathrm{D}^{0} \mathrm{X}$ exciton complex, what is evidenced by decrease of $\mathrm{I}_{\mathrm{D} 0 \mathrm{X}} / \mathrm{I}_{\mathrm{BE}}$ ratio (Fig. 7). Due to bound exciton destruction the donor impurities become available for donation electrons to conduction band, thereby increasing the free carrier concentration as is shown on Fig. 6. The most probable donor impurities, according to the SIMS results (Fig. 8) may be aluminum, silicon or hydrogen. However, the question arises as to whether all potential donor impurities can affect both the optical and the electrical processes.

In order to understand which of impurities plays a role of donor we define the localization energy of $\mathrm{D}^{0} \mathrm{X}$ exciton from PL results and compare it with well-known experimental findings provided by Meyer et al [32].

The bound exciton localization energy can be determined using the following relation between the energy of free exciton and $\mathrm{D}^{0} \mathrm{X}$ energy [31]:

$$
E_{\mathrm{loc}}=E_{\mathrm{FX}}(T)-E_{\mathrm{D} 0 \mathrm{X}}(T)
$$

Our calculations show that the exciton localization energy is about $16.3 \mathrm{meV}$. It is much smaller than the thermal energy at room temperature $k_{\mathrm{B}} T=25 \mathrm{meV}$ (that's why the destruction of bound exciton is observed in the high temperature mode). According to the Haynes rule the donor binding energy and exciton localization energy are related as [31]:

$$
E_{\mathrm{d}}=E_{\mathrm{loc}} / \beta
$$

where $\beta$ is a constant and estimated to be 0.3 for $n$-type semiconductors. Therefore, the value of the donor binding energy is equal to $54.3 \mathrm{meV}$. It should be mentioned that we used two different terms: donor activation energy and donor binding energy, which are not the same. Typically, the donor activation energy in semiconductor physics is determined as the minimum amount of energy which electrons of donor impurity must to receive in order to get into the conduction band. For the $n$-type semiconductor, one can write the following equation:

$$
E_{\mathrm{act}}=1 / 2\left(E_{\mathrm{c}}-E_{\mathrm{d}}\right)
$$

where $E_{\mathrm{d}}$ is the donor level, $E_{\mathrm{c}}$ is the conduction band energy. Meanwhile, in the frames of the concept of the effective mass approximation the energy difference between the ground state neutral bound exciton and excited state neutral bound exciton can be regarded as a donor binding energy To identify the type of donor impurity let us consider the role of all potential donor impurities separately.

\section{i) Silicon}


Silicon is an element of group IV and may play a role both a donor and an acceptor. Substituting the zinc in cation sublattice of $\mathrm{ZnO}$ matrix or forming the corresponding interstitial defect the silicon is donor impurity, and vice versa, when silicon substitutes the oxygen ions in the anion sublattice then it is the acceptor-type dopant. Hall measurements indicate that the grown films were characterized by electronic conductivity. This indicates that silicon impurity can be donor-type and can donate a free electron. Furthermore, an increase in free electron concentration due to the silicon doping has been reported (before reaching the thermodynamic solubility limit of silicon in zinc oxide) $[33,34]$. However, there are no reliable data on the effect of silicon on the formation of bound excitons in zinc oxide.

\section{ii) Aluminum}

Aluminum is the most likely candidate for the role of donor impurity, because of the calculated donor binding energy [32] for zinc oxide. On the other hand, it should be noted that Meyer et al. reported that the $\mathrm{I}_{8}$ exciton line $(3.3598 \mathrm{eV})$ as caused by a gallium. In our case $\mathrm{Ga}$ was not observed by the SIMS analysis, therefore this line cannot be attributed to Ga impurity. Therefore, Al impurity is the most probable origin for the observed PL line.

\section{iii) Hydrogen}

Photoluminescence spectra did not reveal the presence of $I_{4}$ exciton line $(3.3628 \mathrm{eV})$, which is often attributed to exciton localized on the $\mathrm{H}$ impurity. In addition, binding energy proper to this impurity (43 meV) does not match with our previous results. Therefore, a decisive role of hydrogen in the formation of radiative recombination channels can be ruled out. However, asymmetric nature of $\mathrm{D}^{0} \mathrm{X}$ band with well-pronounced shoulder in the high-energy part of the spectrum may indicate an overlap of excitonic lines associated with different donor impurities. Therefore, we do not exclude completely the availability of $\mathrm{H}$ in our sample or its overall effect on the material properties.

Despite the fact that PL studies have shown the presence of only shallow donors (but not shallow acceptors) in $\mathrm{ZnO}$ film, electrical transport is mainly determined by the ratio between shallow donors and compensating acceptors. If the concentration of shallow donors is less than the number of acceptors, the shallow donors are ionized at almost any temperature values. In this case, the film will be characterized by low values of mobility and conductivity. On the other hand, if the donor concentration exceeds the concentration of acceptors, the shallow donors will be easier to give free electrons, thereby improving electrical properties. In this case, according to SIMS results, the most probable acceptors can be potassium, sodium and silicon (at the substitution of oxygen). Moreover, the concentration of potassium and sodium is even higher than the concentration of $\mathrm{Al}$ donor impurity. However, $\mathrm{Na}$ and $\mathrm{K}$ are acceptors, accordingly to 
the recent studies $[35,36]$. Taking into account that the grown films have electronic conductivity and are nearly degenerate semiconductors on can assume that a crucial role in the $n$-conductivity play the intrinsic point defects, silicon and hydrogen. We have depicted the temperature evolution of the main optical transitions in $\mathrm{ZnO}$ films (Fig. 9). We believe that Al plays an important role, creating the donor level $\mathrm{D}^{\mathrm{Al}}$, which is thermally ionized with temperature increase, resulting in the dominant FX emission at room temperature.

\section{Conclusions}

It is revealed, that the PL spectra at low temperature range $(4-80 \mathrm{~K})$ are dominated by intense $\mathrm{D}^{0} \mathrm{X}$ line at $3.3599 \mathrm{eV}$ (at $4 \mathrm{~K}$ ), which is attributed to the presence of $\mathrm{Al}$ as a neutral impurity in $\mathrm{ZnO}$. This is evidenced by the value of donor binding energy, calculated from PL spectra as peculiar for Al, as well as SIMS data, revealing the presence of Al as impurity. The correlation of temperature dependent PL and Hall Effect measurements is revealed in terms of carrier mobility and the intensity of $\mathrm{D}^{0} \mathrm{X}$ line. Particularly, the temperature activated dissociation of bound exciton, attributed to decrease of $\mathrm{D}^{0} \mathrm{X}$ line is observed to occur simultaneously with the increase of the carrier's mobility. This we explain as aluminum activation as a donor and respective providing the free electron to the conduction band. The thermal activation energy $E_{\mathrm{D}}=20 \mathrm{meV}$ determined from the Hall data is in agreement with the optical activation energy, which is derived to be $\sim 20 \mathrm{meV}$ by temperature-dependent PL spectra. It is revealed that the exciton localization energy is $16.3 \mathrm{meV}$, what agrees well with localization energy of $15.3 \mathrm{meV}$ for Al impurity reported by other authors (e.g. Ref. [32]). Furthermore, the calculated value of donor binding energy $54.3 \mathrm{eV}$ is also in agreement with ionization energy of $53 \mathrm{meV}$ mentioned in earlier reports for aluminum in $\mathrm{ZnO}$ films. Thus, our results prove that the commonly observed line at $3.3599 \mathrm{eV}$ on low temperature PL spectra of $\mathrm{ZnO}$ is a neutral donor bound exciton emission due to $\mathrm{Al}$ impurity.

\section{Acknowledgements}

This publication is part of Dr. I. Shtepliuk's research work at Linkoping University, thanks to a Knut and Alice Wallenberg Foundation. Dr. V. Khranovskyy acknowledges the Ångpanneföreningens Forskningsstiftelse (Grant 14-517) for the support of this work.

\section{References}

[1] D. C. Look, B. Claflin, Ya. I. Alivov, S. J. Park, Phys. Status Solidi A $201 \quad$ (2004) 22032212.

[2] Z. C. Feng, Handbook of $\mathrm{ZnO}$ and related materials, Taylor and Francis/CRC Press, Florida, 2012.

[3] U. Ozgur, D. Hofstetter, H. Morkoc, Proc. IEEE 98 (2010) 1255 - 1268. 
[4] I. Shtepliuk, V. Khranovskyy, G. Lashkarev, V. Khomyak, V. Lazorenko, A. Ievtushenko, M. Syväjärvi, V. Jokubavicius and R. Yakimova, Solid State Electron. 81 (2013) 72-77.

[5] I. Shtepliuk, V. Khranovskyy, G. Lashkarev, V. Khomyak, A. Ievtushenko, V. Tkach, V. Lazorenko, I. Timofeeva, R. Yakimova, Appl. Surf. Sci. 276 (2013) 550- 557.

[6] G. F. Neumark, I. L. Kuskovsky, H. Jiang, Wide Bandgap Light Emitting Materials and Devices, Wiley-VCH Verlag, Weinheim, 2007.

[7] I. Shtepliuk, Superlattices Microstruct. 71 (2014) 62-71.

[8] I. Shtepliuk, V. Khranovskyy, and R. Yakimova, Thin Solid Films (2015), http://dx.doi.org/10.1016/j.tsf.2015.02.001.

[9] O. Melikhova, J.Č́žžek, I. Procházka, R. Kužel, M. Novotný, J. Bulíř, J. Lančok2, W. Anwand, G. Brauer, J. Connolly, E. McCarthy, S. Krishnamurthy, J.-P. Mosnier, J. Phys. Conf. Ser. 443 (2013) 012018.

[10] M. Syväjärvi, R. Yakimova, H. Jacobsson, and E. Janzen, J. Appl. Phys. 88 (2000) 1407.

[11] M. H. Xie, L. X. Zheng, S. H. Cheung, Y. F. Ng, Huasheng Wu, S. Y. Tong and N. Ohtani, Appl. Phys. Lett. 77 (2000) 1105

[12] V. Khranovskyy, I. Tsiaoussis, G. Yazdi, L. Hultman and R. Yakimova, J. Crystal Growth 312 (2010) 327-332.

[13] B. K. Meyer, H. Alves, D. M. Hofmann, W. Kriegseis, D. Forster, F. Bertram, J. Christen, A. Hoffmann, M. Straßburg, M. Dworzak, U. Haboeck, and A. V. Rodina, Phys. Status Solidi B 241 (2004) 231

[14] V. Khranovskyy, R. Yakimova, Physica B 407 (2012) 1533.

[15] V. Khranovskyy and R. Yakimova, Heteroepitaxy of ZnO on SiC as a route toward nanoscale p-n junction, in: Z. C. Feng (Eds.), Handbook of Zinc Oxide and Related Materials, Vol. 2: Devices and Nano-Engineering, Taylor \& Francis, 2012, pp. 83 - 131.

[16] X. H. Wang and S. J. Xu, Appl. Phys. Lett. 102 (2013) 181909

[17] M. Leroux, N. Grandjean, B. Beaumont, G. Nataf, F. Semond, J. Massies, and P. Gibart, J. Appl. Phys. 86 (1999) 3721

[18] H. P. He, H. P. Tang, Z. Z. Ye, L. P. Zhu, B. H. Zhao, L. Wang, and X. H. Li, Appl. Phys. Lett. 90 (2007) 023104

[19] J. Krustok, H. Collan, and K. Hjelt, J. Appl. Phys. 81 (1997) 1442

[20] D. Sentosa, B. Liu, L.M. Wong, Y.V. Lim, T.I. Wong, Y.L. Foo, H.D. Sun, S.J. Wang, J.Cryst. Growth 319 (2011) 8-12

[21] S.-J. Park, Y.-H. Hwang, H.-K. Kim, J. Korean Phys. Soc. 60 (2012) 466-471.

[22] Y.P. Varshni, Physica 34 (1967) 149. 
[23] P. Misra, T.K. Sharma, S. Porwal, L.M. Kukreja, Appl. Phys. Lett. 89 (2006) 161912.

[24] R. Hellmann, M. Koch, J. Feldmann, S. T. Lundiff, E.O. Gobel, D. R. Yakovler, A. Waag and G. Landwehr, Phys. Rev. B 48 (1993) 2847

[25] M. O’Neill, M. Oestriech, W. W. Ruhle and D. E. Ashenford, Phys. Rev. B 48 (1993) 8980.

[26] Y. Zhang, D.-J. Chen, and C.-T. Lee, Appl. Phys. Lett. 91 (2007) 161911

[27] P. Lantenschlager, M. Garriga, S. Logothetidis, and M. Cardona, Phys.Rev. B 35 (1987) 9174

[28] J. M. Calleja and M. Cardona, Phys. Rev. B 16 (1977) 3753-3761.

[29] K. A. Alim, V. A. Fonoberov and A. A. Balandin, Appl. Phys. Lett. 86 (2005) 053103.

[30] H B Ye, J F Kong, W Z Shen, J L Zhao and X M Li, J. Phys. D: Appl. Phys. 40 (2007) 5588-5591

[31] K. T. Roro, G. H. Kassier, J. K. Dangbegnon, S. Sivaraya, J. E. Westraadt, J. H. Neethling, A. W. R. Leitch and J. R. Botha, Semicond. Sci. Technol. 23 (2008) 055021.

[32] B. K. Meyer, J. Sann, D. M. Hofmann, C. Neumann and A. Zeuner, Semicond. Sci. Technol. 20 (2005) S62-S66

[33]A. K. Das, P. Misra and L. M. Kukreja, J. Phys. D: Appl. Phys. 42 (2009) 165405

[34] R. Schifano, M. Schofield, L. Vines, S. Diplas, E. V. Monakhov and B. G. Svensson, IOP Conf. Ser.: Mater. Sci. Eng. 34 (2012) 012007

[35] P.T. Neuvonen, L. Vines, A. Yu. Kuznetsov, B.G. Svensson, X. Du, F. Tuomisto and A. Hallén, Appl. Phys. Lett. 95 (2009) 242111.

[36] A. Janotti and C. G Van de Walle, Rep. Prog. Phys. 72 (2009) 126501. 


\section{Figures captions}

Figure 1. PL spectra of $\mathrm{ZnO}$ films on $4 \mathrm{H}-\mathrm{SiC}$ substrates at temperature range $10-290 \mathrm{~K}$ : the wide range linear scale (a) and high resolution logarithmic scale plots (b) are shown: the main peaks FX, $\mathrm{D}^{0} \mathrm{X}$, TES and DAP are marked.

Figure 2. Temperature dependence of the integrated PL intensity for the $\mathrm{ZnO}$ films grown on 4H-SiC substrates. Squares are the experimental data and solid lines are the fit with Eq. (1). The estimated activation energy in high temperature regime is $20 \mathrm{meV}$ and in the low temperature regime is $7.5 \mathrm{meV}$.

Figure 3. The temperature dependent energy position of the peaki for the $\mathrm{D}^{0} \mathrm{X}$ and $\mathrm{FX}$ emission bands. The black and red circles are the experimental data and the lines are their respective fitting by Varshni's formula.

Figure 4. $\mathrm{PL}$ emission linewidth as a function of temperature for $\mathrm{ZnO}$ films. Red circles are the experimental data and the solid lines are the least-squares fit of data by Eq. (3).

Figure 5. Image of the resistance depth profile of $\mathrm{ZnO}$ film on $4 \mathrm{H}-\mathrm{SiC}$ substrate, obtained by Scanning Spreading Resistance Measurements (SSRM). The respective areas of ZnO film and SiC substrate are shown.

Figure 6. Temperature dependence of the electron concentration for $\mathrm{ZnO}$ film grown onto $4 \mathrm{H}$ $\mathrm{SiC}$ substrate (a) and temperature dependence of the compensation ratio (b).

Figure 7. Temperature dependence of $\mathrm{I}_{\mathrm{D} 0 \mathrm{X}} / \mathrm{I}_{\mathrm{BE}}$ ratio, obtained from the PL measurements.

Figure 8. SIMS data of the different impurities in $\mathrm{ZnO}$ films on $\mathrm{SiC}$ substrate: the concentration versus depth profiles are shown.

Figure 9. Schematic representing the optical transitions in $\mathrm{ZnO}$ with $\mathrm{Al}$ unintentional dopant and the free electrons density and their respective temperature evolution. 


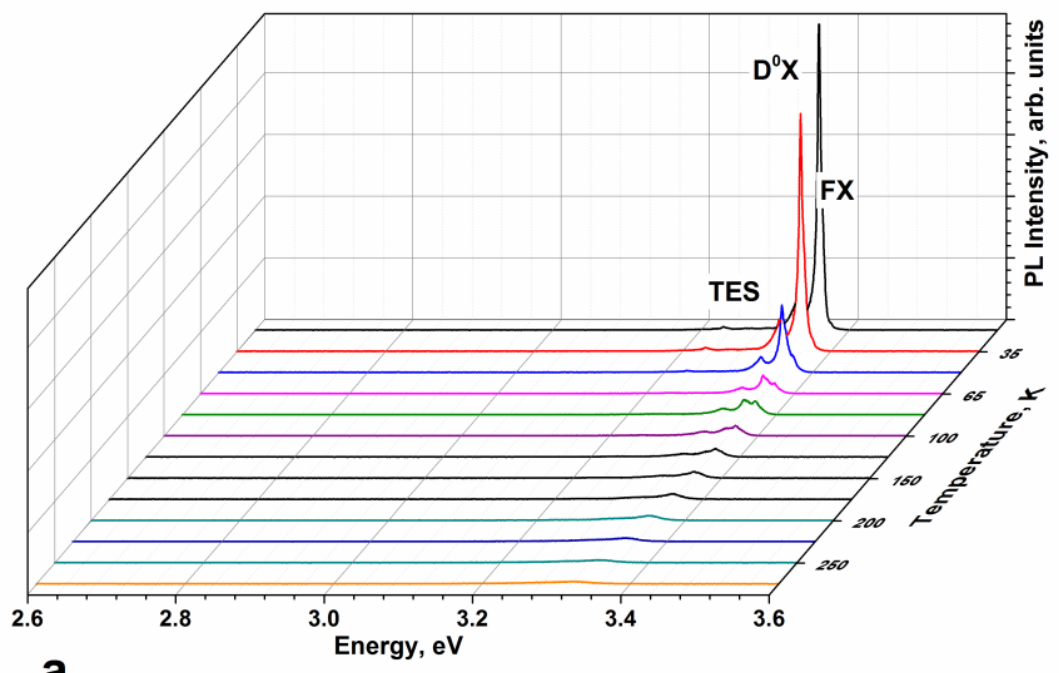

a

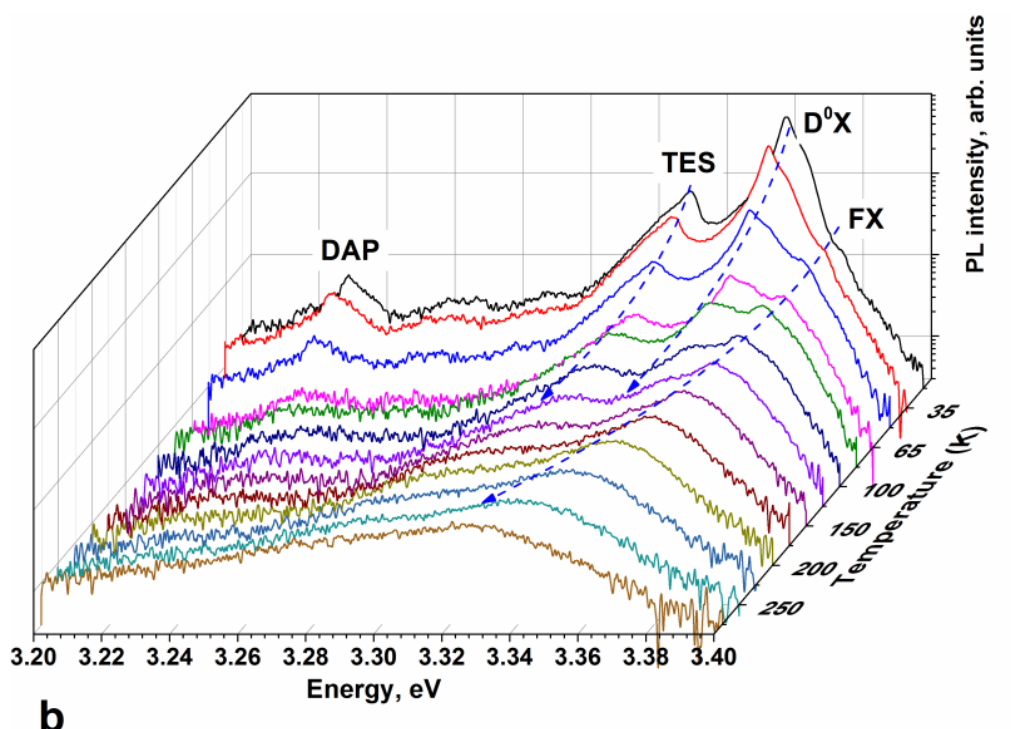

Figure 1 


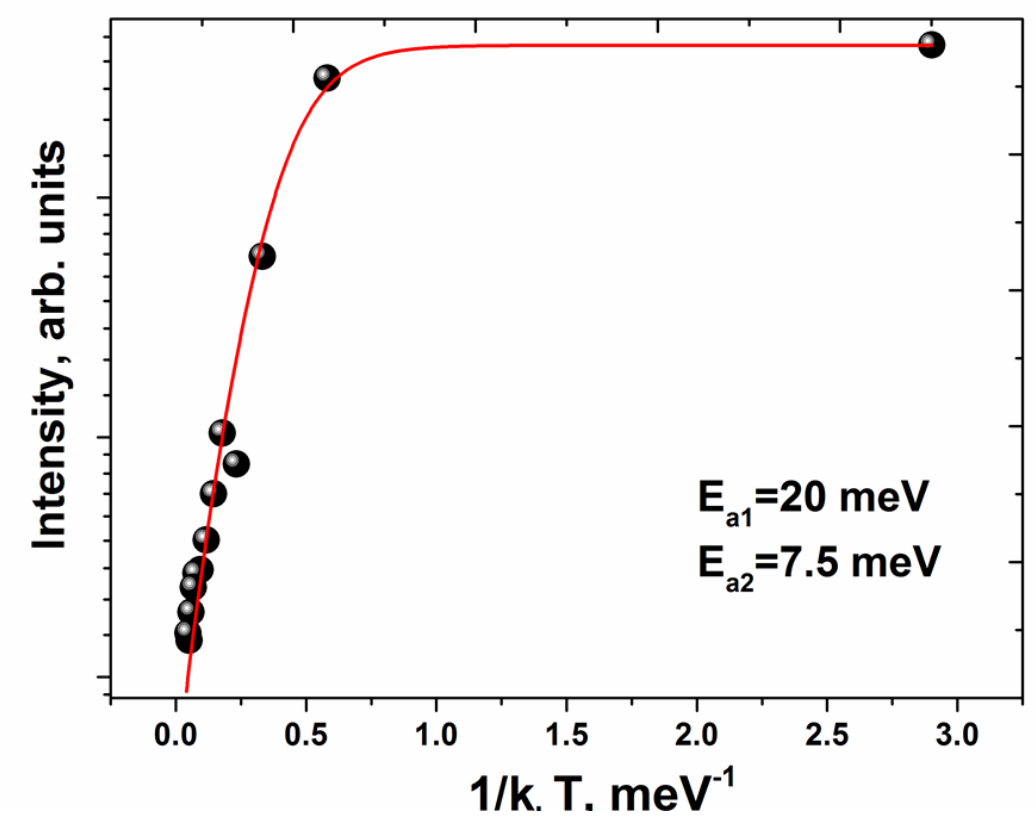

Figure 2.

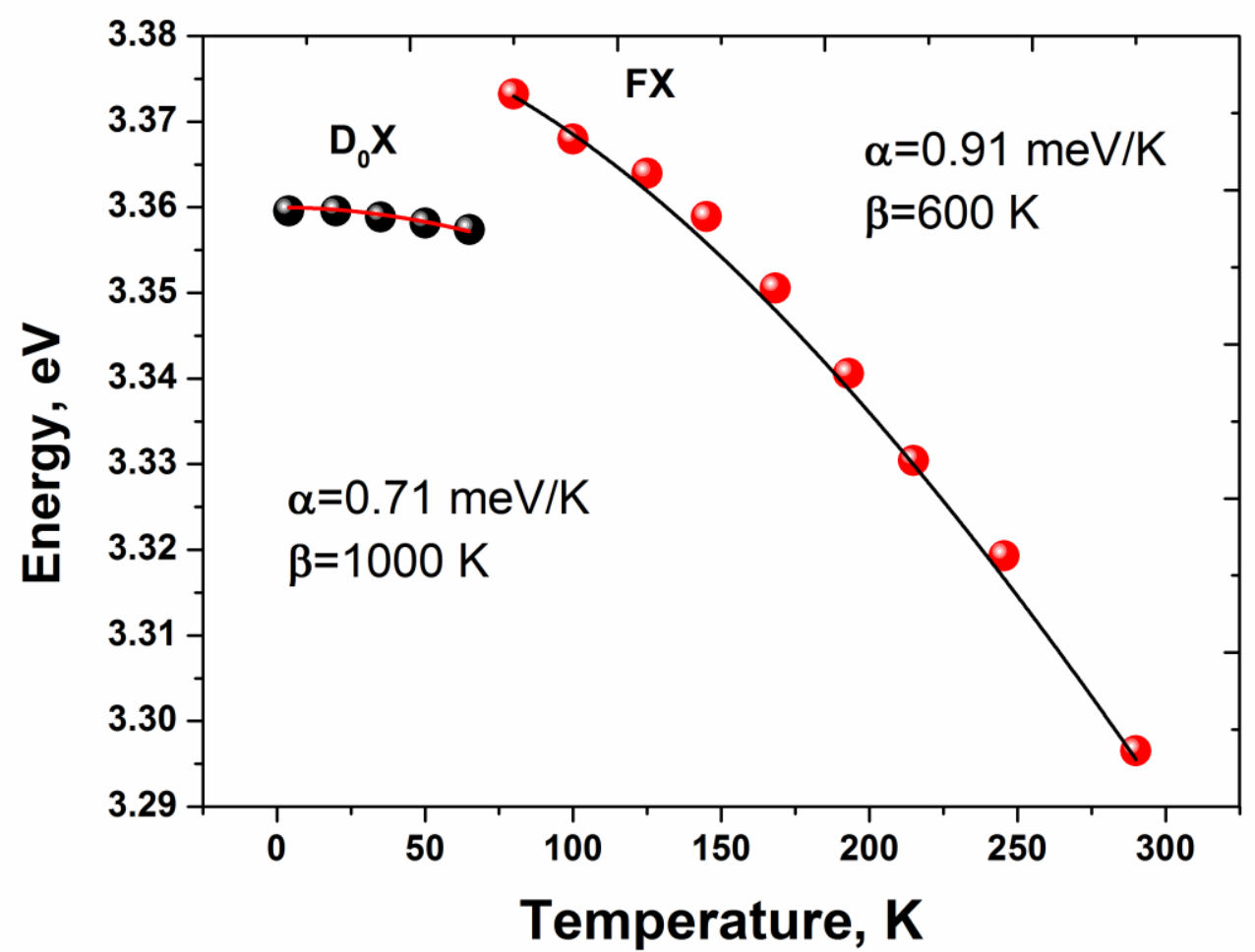

Figure 3 


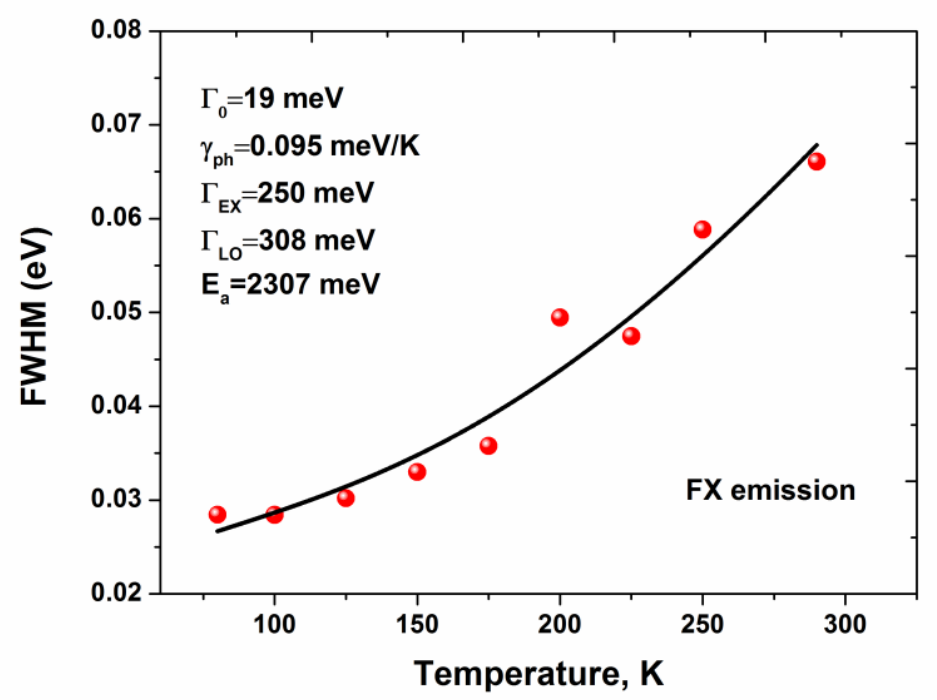

Figure 4.

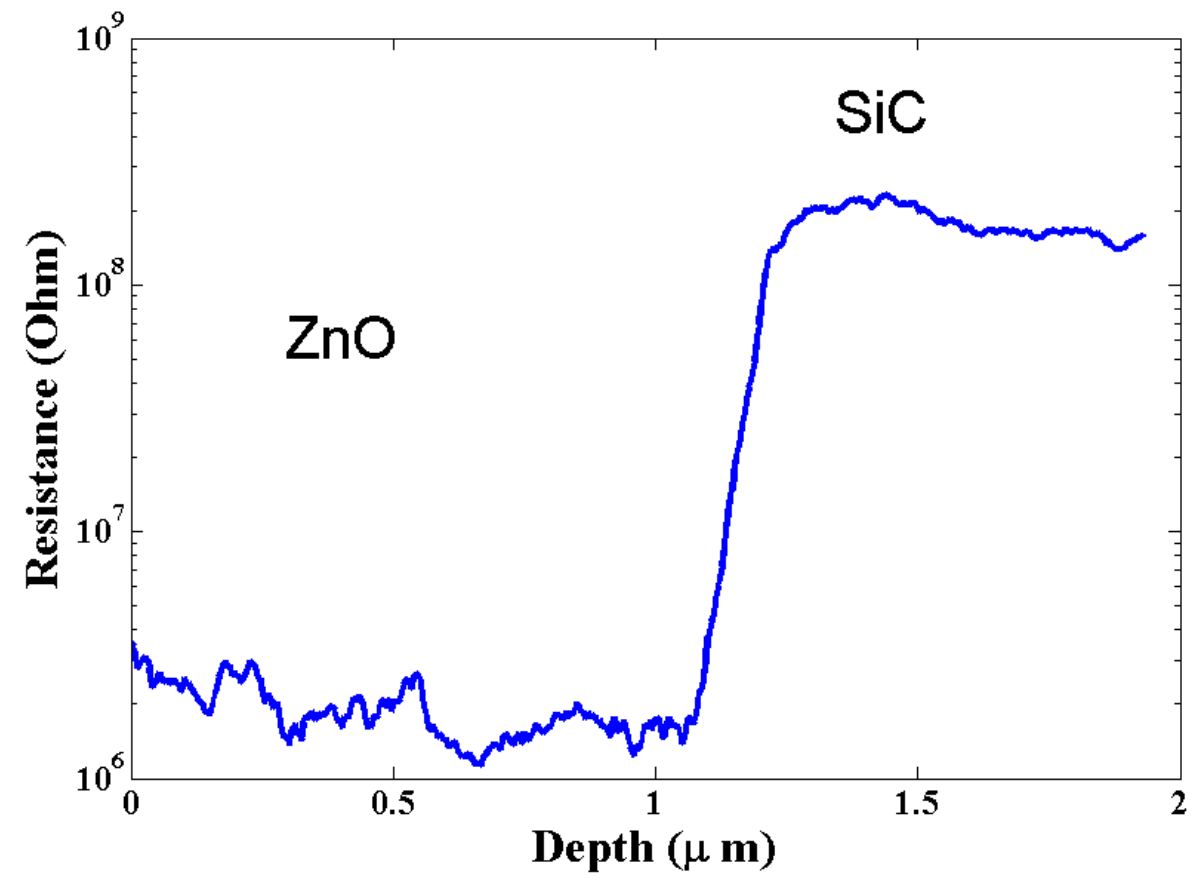

Figure 5. 

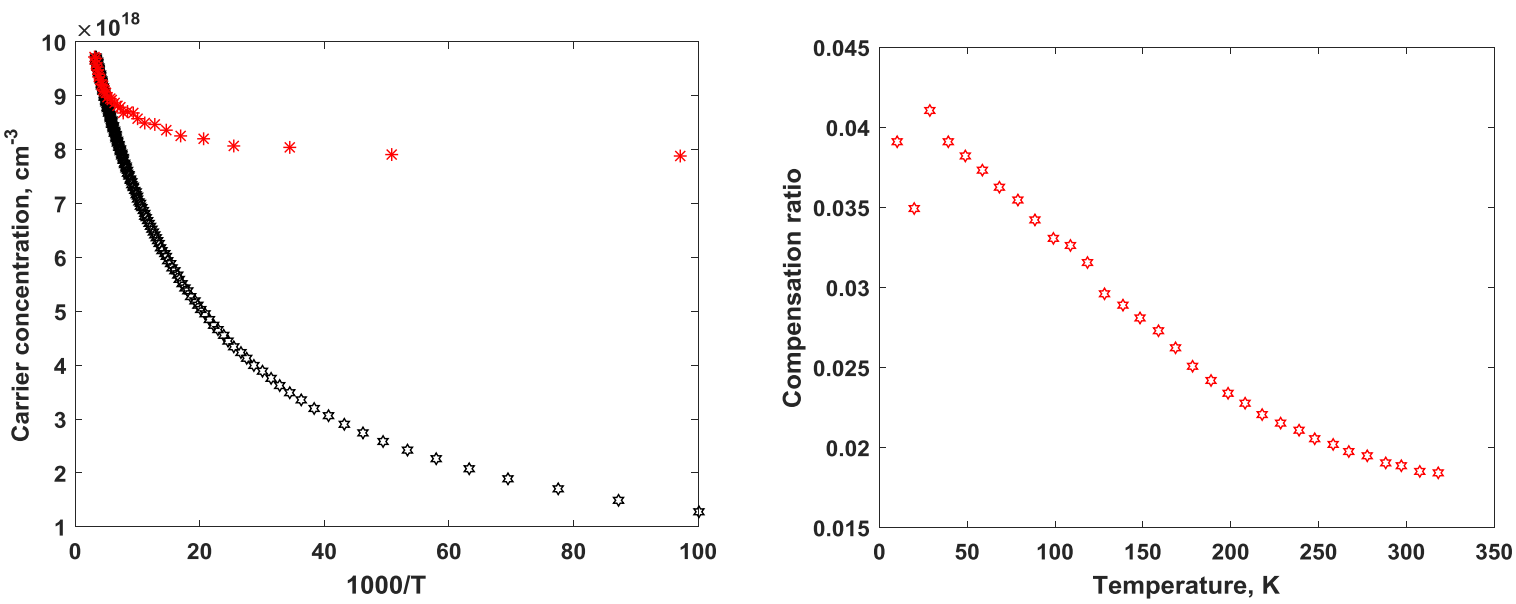

Figure 6.

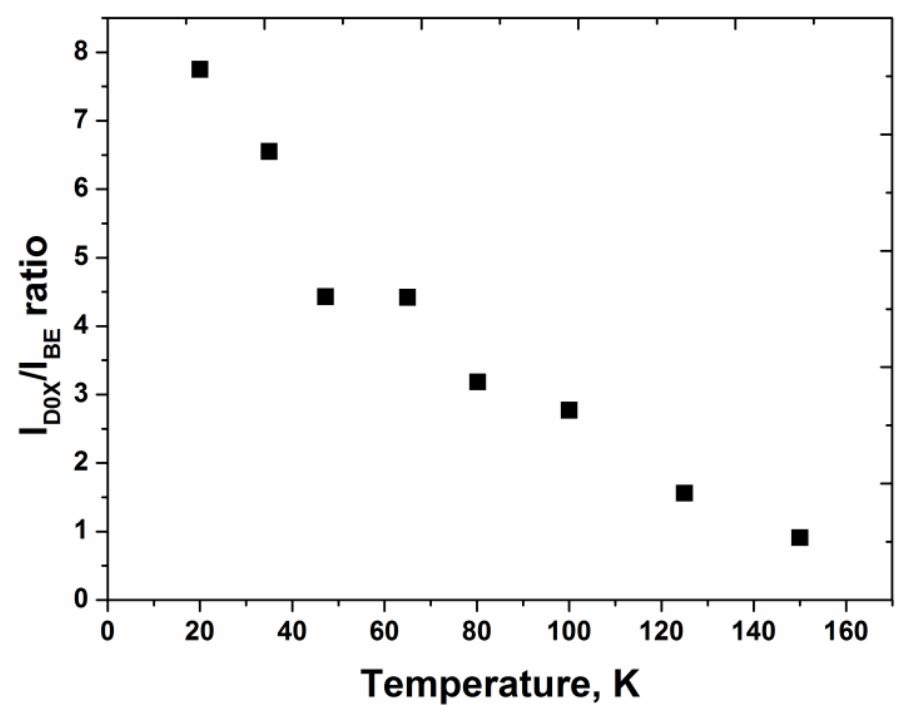

Figure 7. 


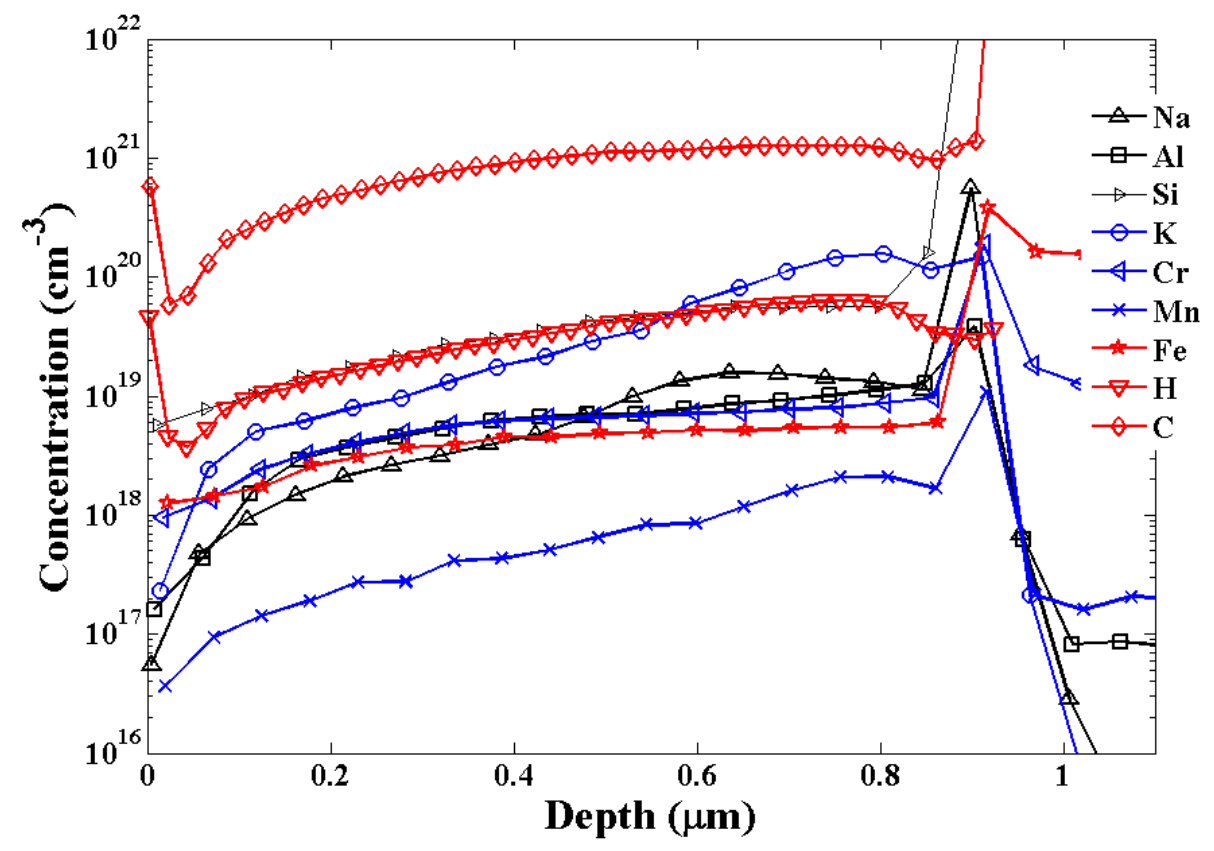

Figure 8.

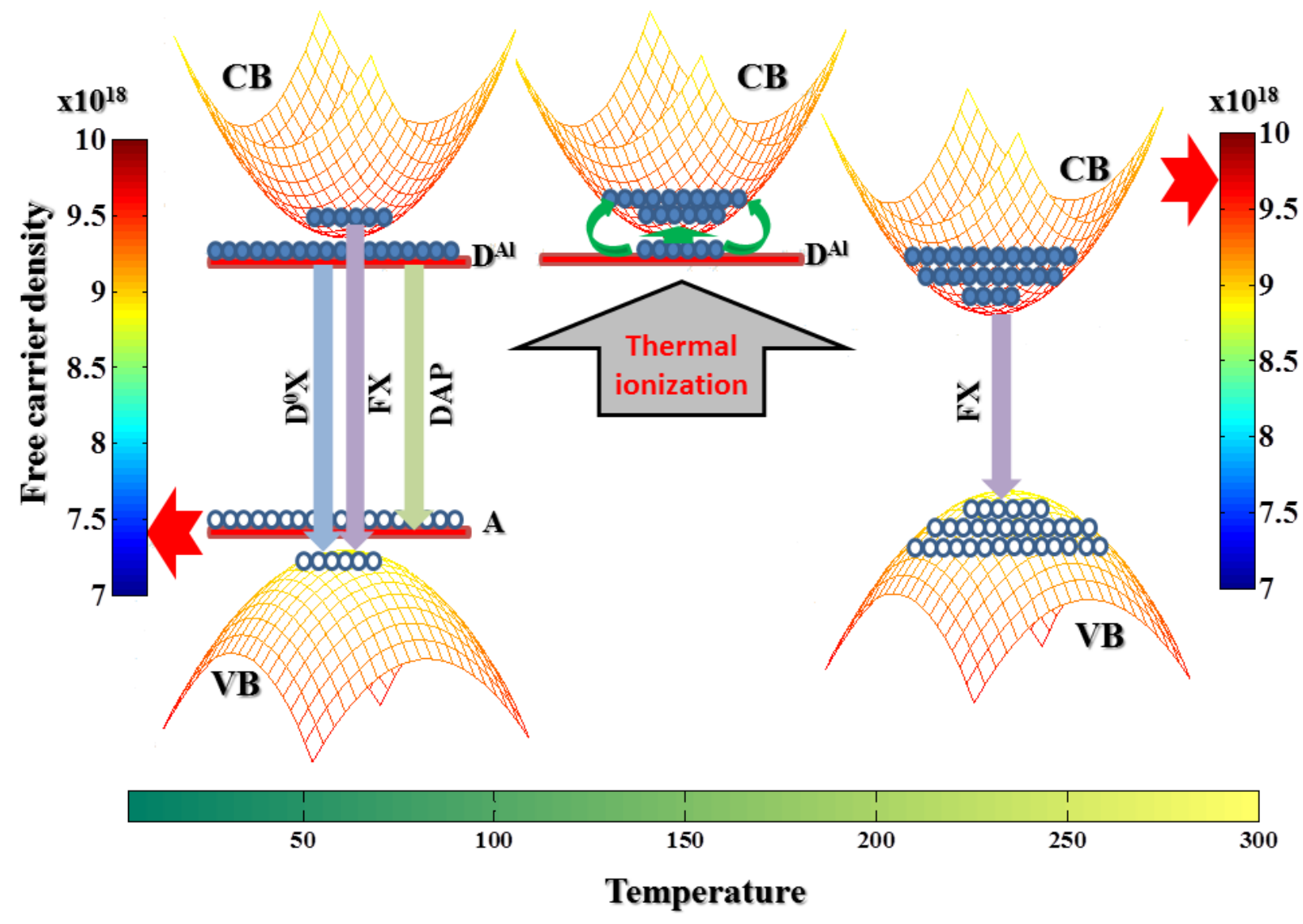

Figure 9. 